\title{
Fabella and patella variants: radiographic prevalence, distribution and clinical relevance in a population of black African descent
}

\author{
Joseph Adetola Adedigba ${ }^{1}$, Bukunmi Michael Idowu ${ }^{2}$, Sarah Perou Hermans ${ }^{1}$, \\ Onoja Frederick Okwori ${ }^{3}$, Stephen Olaoluwa Onigbinde ${ }^{4}$, Kehinde S. Oluwadiya ${ }^{5}$, \\ Angela Ameyaa Amoako ${ }^{6}$, Mandy C. Weidenhaft ${ }^{7}$ \\ ${ }^{1}$ Department of Radiology, Tulane University School of Medicine, New Orleans, LA, USA, ${ }^{2}$ Department of Radiology, Union Diagnostics and Clinical \\ Services, Lagos, Nigeria, ${ }^{3}$ Department of Epidemiology, Tulane University School of Public Health and Tropical Medicine, New Orleans, LA, USA, \\ ${ }^{4}$ Department of Anatomical sciences, School of Medicine, St George's University, Grenada, West Indies, ${ }^{5}$ Department of Surgery, Ekiti State University, \\ Ado-Ekiti, Nigeria, ${ }^{6}$ Department of Microbiology, University of Wisconsin-La Crosse, La Crosse, WI, ${ }^{7}$ Department of Radiology, Tulane University \\ School of Medicine, New Orleans, LA, USA
}

\begin{abstract}
To evaluate the radiographic characteristics and prevalence of fabella and patella variants in an indigenous African population. This retrospective observational study of orthogonal knee radiographs of 377 consecutive subjects was conducted in Lagos, Nigeria, from February 2017 to November 2017. The presence of bipartite/multipartite patella, as well as the presence of fabella were noted. The craniocaudal diameter, anteroposterior diameter, fabello-femoral distance and fabello-tibial distance of the fabella were measured. $P \leq 0.05$ represented a statistically significant result. Three hundred and seventy-seven subjects were enrolled. The average age was $41.22 \pm 21.37$ years with a range of 3-100 years old. There were 158 male (41.9\%) and 219 female (58.1\%) subjects. The prevalence of fabella was $11.94 \%$. There was a positive correlation between age $<47$ and $\geq 47$ and occurrence of fabella, $P<0.015$. There was no statistically significant difference between the mean male and female measured fabella diameters. The overall prevalence of bipartite and multipartite patella in this study was $2.12 \%$. Among male and female subjects, the difference in prevalence of bi and multipartite patella was statistically significantly, $P=0.03$. The prevalence of fabella and patella variants was lower in this study compared to the findings in other populations and ethnicities. Sex and age were significantly correlated with fabella prevalence. The results reported in this study will facilitate future studies examining the correlations between fabella and patella variants and various knee pathologies in a population of Black African descent.
\end{abstract}

Key words: Sesamoid bones, Knee, Prevalence, Africa South of the Sahara, Diagnostic imaging

Received August 13, 2020; Revised January 4, 2021; Accepted January 8, 2021

\author{
Corresponding author: \\ Joseph Adetola Adedigba (iD \\ Department of Radiology, Tulane University School of Medicine, New \\ Orleans, LA 70112, USA \\ E-mail: jaadedigba@gmail.com
}

\section{Introduction}

Sesamoid bones are small round bones embedded in tendons. They are found near the joints of the upper and lower extremities [1]. Examples of sesamoid bones include the patella, fabella, Os acromiale, Os acetabuli, Os trigonum, Hallux sesamoids, and many others. The patella is the largest sesamoid bone in the body. It is held in place by the quadriceps and patella tendons. The patella protects the anterior 
surface of the knee joint. It increases the lever arm of the extensor mechanism allowing for more effective knee flexion [2]. At birth it is made of cartilage and begins to ossify between 2 to 6 years of age [3]. During adolescence, secondary ossification centers of the patellar bone usually fuse to form a single ossification center, therefore forming a single bone in most of the population [4]. In a small percentage of people, a failure of fusion of the ossification centers on the patella leads to a bipartite or tripartite patella [2]. The bipartite patella was first described by Gruber [5] in 1883 and later classified by Saupe [6] in 1921 into three subgroups based on the location of the fragments. The Saupe classification was reviewed in 2010 by Oohashi et al. [7], who reported more cases of, and presented a new classification of multipartite patella [8]. Multipartite patella anomaly has been reported as a cause of anterior knee pain and symptomatology $[8,9]$. In addition, knowledge of multipartite patella will help in the differentiating of patella fractures and avulsions from normal variants [2].

The fabella is a small sesamoid bone embedded in the tendon of the lateral head of the gastrocnemius muscle which articulates with the lateral femoral condyle. It is made up of cartilage or ossified bone and is found in 10\% to $30 \%$ in certain populations [10]. The fabella first appears as an

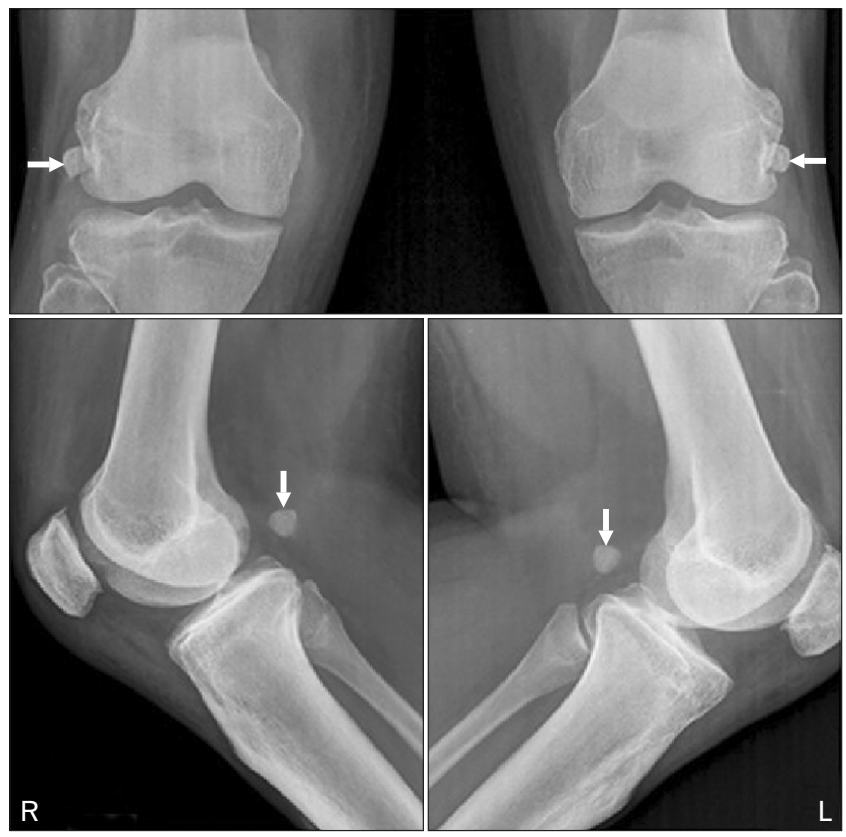

Fig. 1. A 40-year-old female with bilateral fabella. Plain anteroposterior and lateral radiographs of both knees showing bilateral fabella sesamoids (arrows). island of cartilage at age 10 and ossifies at age 12 to 15 years [11]. Studies investigating populations of Asian descent have noted increased incidence of the fabella, with one Japanese cadaver study of 212 knees reporting an incidence of $85.8 \%$, and a Chinese cadaver and radiography study of 61 knees reporting an incidence $86.9 \%[12,13]$. This high incidence has been speculated to be due to kneeling and squatting in some Asians, which increases the pressure on the lateral femoral condyle leading to fabella development and ossification [14]. Fabellae can be either bony or cartilaginous and are usually asymptomatic. They are found incidentally on radiographs (bony fabella), computed tomography (bony fabella), and magnetic resonance imaging (bony and cartilaginous fabella). Occasionally, various pathologies of the fabella may cause atypical pain often mistaken for a more common cause of clinical symptoms such as an osteophyte or an intra-articular loose body [15]. Clinically significant pathologies of the fabella include fracture (from trauma or stress fracture secondary to total knee arthroplasty), dislocation, degeneration (chondromalacia fabellae/fabella syndrome), symptomatic

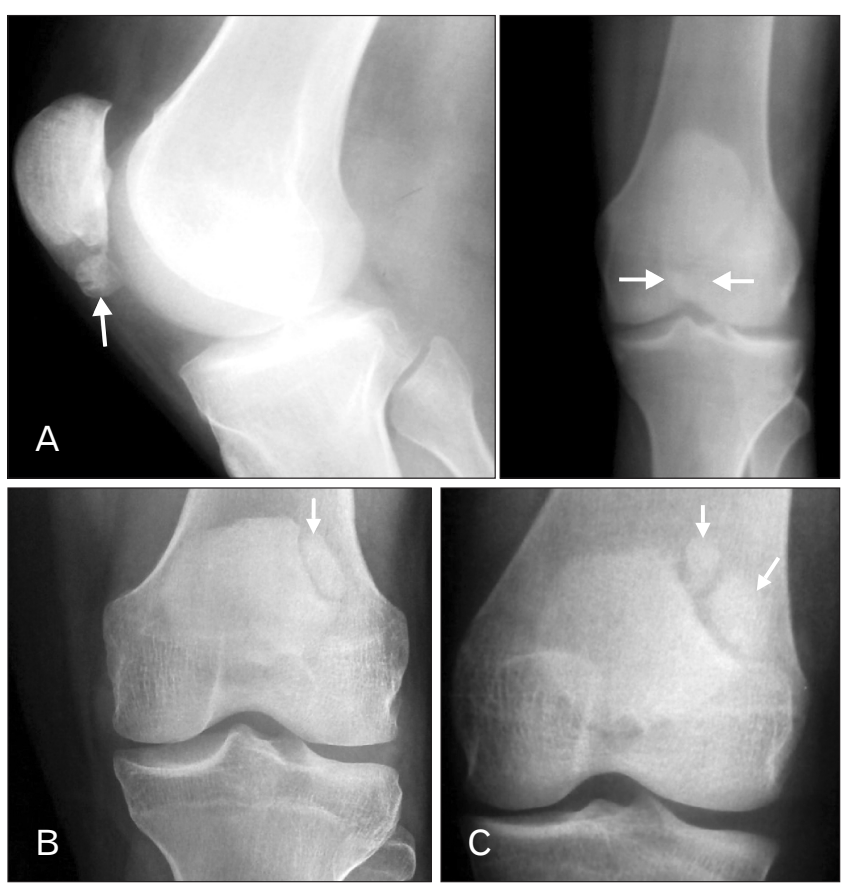

Fig. 2. (A) A 30-year-old male. Plain lateral and anteroposterior radiographs of the knee showing a type I (inferior margin) bipartite patella (arrows), according to the Saupe classification. (B) A 30-yearold male. Plain lateral and anteroposterior radiographs of the knee showing a type I (inferior margin) bipartite patella (arrow), according to the Saupe classification. (C) Plain anteroposterior radiograph of the knee showing a type III (superolateral margin) tripartite patella (arrows), according to the Saupe classification. 
(painful) enlargement, and association with osteoarthrosis [16-19]. Understanding the radiological characteristics of fabella and patella variants is vital to diagnosing the sequela and pain syndromes that may result from this anatomical variant.

The imaging prevalence and clinical relevance of these anatomic variations are understudied in Black African populations. Currently, to the best of our knowledge, there are no imaging studies describing the prevalence of patella variants and fabella in a Black African population. The only study in sub-Saharan Africa described the prevalence of fabella in cadavers [20]. Most of the previous studies on the imaging prevalence of patella variants and fabella involved Caucasian populations, in Europe and North America, and Asian populations across the far East $[9,14,21-25]$. In addition, there is currently no study in the literature, to the best of our knowledge, that examines the prevalence of patella variants and fabella simultaneously in the same study population. This combined study allows us to predict the likelihood of having both anatomical variations in the same subjects and to describe the relative prevalence of patella variants and fabella since they are being evaluated in the same study population.

\section{Materials and Methods}

This was a retrospective observational study of orthogonal knee radiographs of 377 consecutive individuals (bilateral

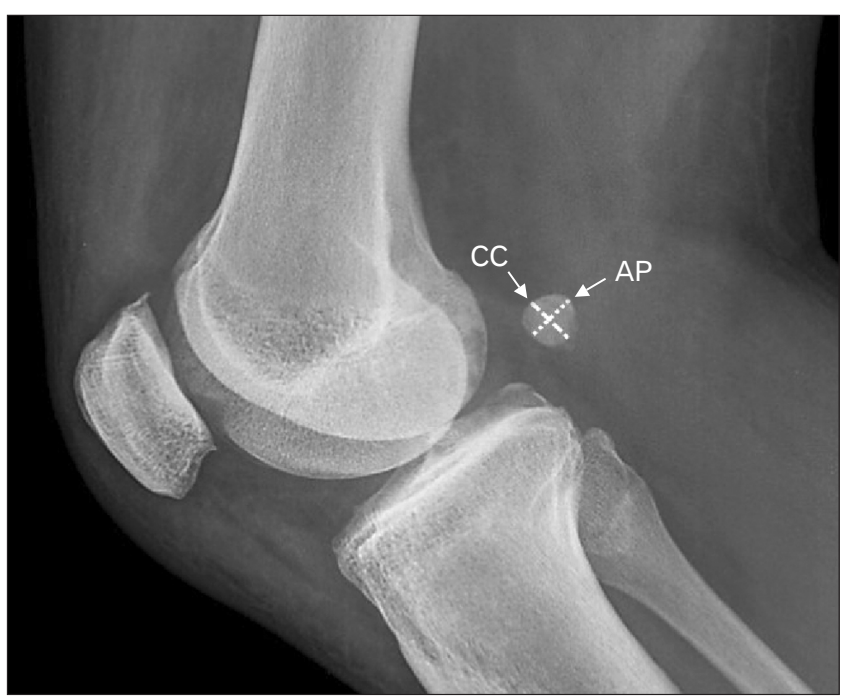

Fig. 3. A 41-year-old female. Plain lateral radiograph of the knee showing measurements of the CC and AP diameters of the fabella. AP, anteroposterior; CC, craniocaudal. knees $=754$ knees) conducted at an out-patient radiodiagnostic center in Lagos, Nigeria, from February 2017 to November 2017. Exclusion criteria included poor image quality/ technical inadequacy, age $<3$ years, presence of comminuted fracture, presence of metallic prosthesis, presence of large osteophytes, congenital/acquired bone deformities, and radiographic evidence of knee joint effusion or popliteal mass. The study protocol conformed to the ethical guidelines of the 1964 Declaration of Helsinki and its subsequent editions. There was a waiver of an ethical committee review on account of the de-identified retrospective nature of the data. The radiographs had been taken using standard exposure factors and a 30 to 45 degrees flexion for the lateral view [26]. The presence of bipartite/multipartite patella, as well as the presence of fabella (Fig. 1), were noted. Bipartite fabella was classified using the Saupe classification; type I: inferior margin bipartite/multipartite patella; type II: direct lateral margin or vertical bipartite/multipartite patella; and type III: superolateral margin bipartite/multipartite patella as shown in Fig. 2 [6].

The craniocaudal diameter (CCD) and anteroposterior diameter (APD) of the fabella were measured on the lateral view (in millimeters), as shown in Fig. 3. The laterality of fabella (unilateral or bilateral) and symmetry of bilateral fabella (symmetric or asymmetric) were documented. Symmetric fabella had the same CCD and APD bilaterally, while asymmetric bilateral fabellae had different values of one or

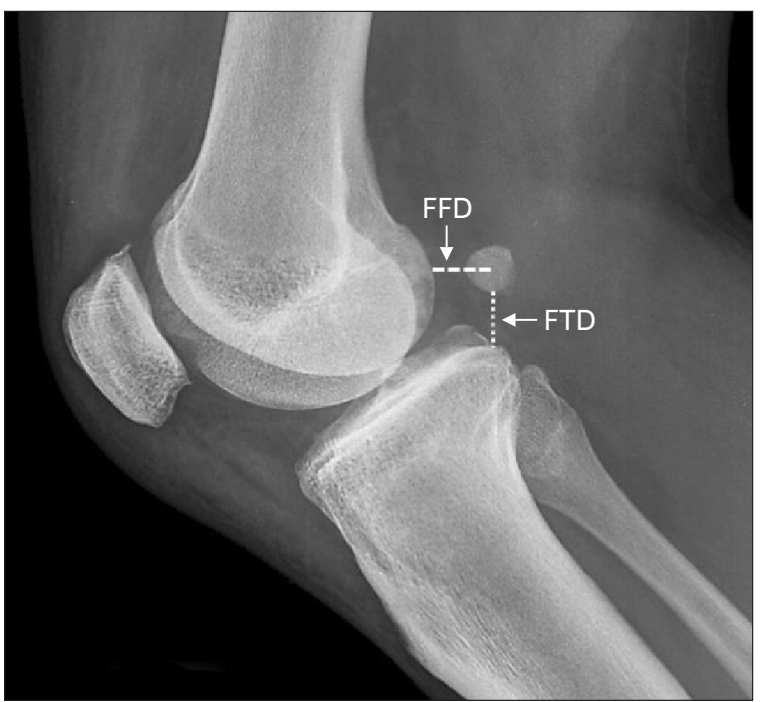

Fig. 4. A 41-year-old female. Plain lateral radiograph of the knee showing measurements of the FFD and FTD. FFD, fabello-femoral distance; FTD, fabello-tibial distance. 

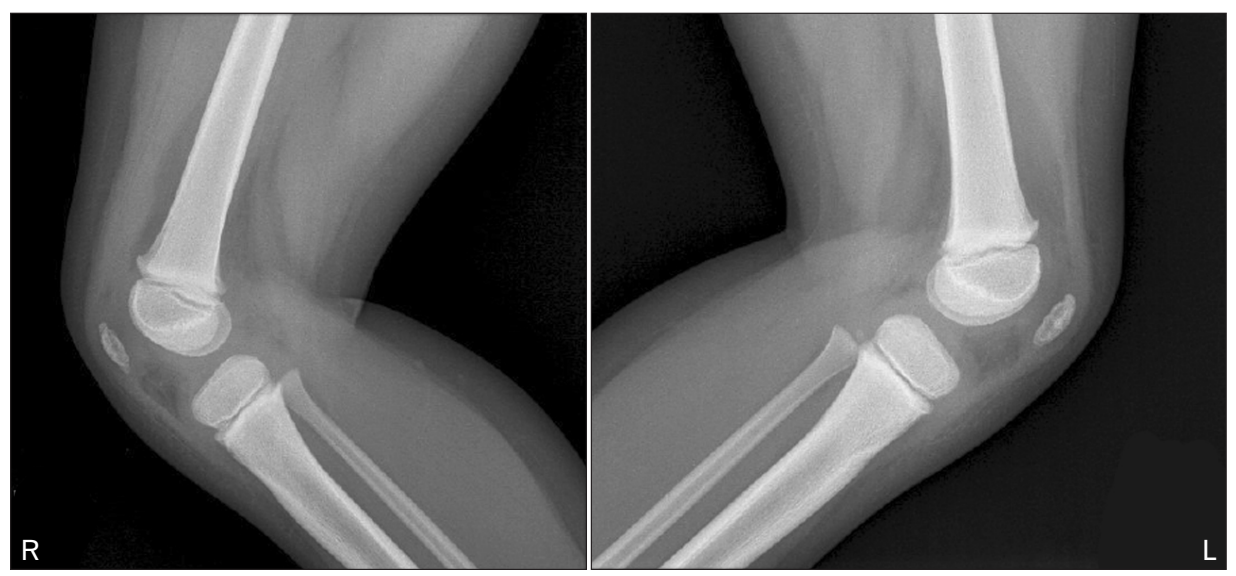

Fig. 5. A 4-year-old male. Plain lateral radiographs of both knees showing 'just ossifying' patellae.

Table 1. Crosstabulation of fabella laterality with sex

\begin{tabular}{lccccc}
\hline \multirow{2}{*}{ Sex } & \multicolumn{4}{c}{ Fabella laterality } & \multirow{2}{*}{ Total } \\
\cline { 2 - 5 } & No fabella & Right & Left & Bilateral & \\
\hline Male & $135(85.4)$ & $6(3.8)$ & $2(1.3)$ & $15(9.5)$ & 158 \\
Female & $197(90.0)$ & $3(1.4)$ & $2(0.9)$ & $17(7.8)$ & 219 \\
Total & $332(88.1)$ & $9(2.4)$ & $4(1.1)$ & $32(8.5)$ & 377 \\
\hline
\end{tabular}

Values are presented as number (\%).

\section{both diameters.}

The fabello-femoral distance (FFD) and the fabello-tibial distance (FTD) were measured on the lateral view, as illustrated in Fig. 4. The FFD was taken as the shortest line ( $\mathrm{mm})$ between the center of the fabella and the posterior edge of the lateral femoral condyle (on the lateral knee radiograph, the lateral femoral condyle is the higher of the two condyles). The FTD was the shortest line $(\mathrm{mm})$ between the lower edge of the fabella and the top of the lateral tibial plateau (Fig. 4) [11]. To minimize interobserver variability, only one staff radiologist interpreted all the radiographs and obtained all measurements. Each measurement was taken thrice, and the average value was recorded, to reduce intraobserver variability.

\section{Statistical analysis}

Descriptive characteristics were majorly categorical and were described by frequencies and proportions. In patients with a fabella, laterality, symmetry (bilaterally equal sizes) and asymmetry (bilaterally unequal sizes) were recorded and analyzed in regard to participant's age and sex. Patella configuration was recorded and compared by age and sex. Four fabella diameters- CCD, APD, FTD, FFD- were recorded and were compared by laterality and sex. Statistical analysis was performed using Pearson's chi-square test for qualitative data and $t$-test for quantitative data. The validity of chi-square
Table 2. Crosstabulation of age and presence of fabella

\begin{tabular}{lccc}
\hline Age group & No fabella & Fabella present & Total \\
\hline $0-19 \mathrm{yr}$ & $74(98.7)$ & $1(1.3)$ & 75 \\
$20-29 \mathrm{yr}$ & $32(100.0)$ & $0(0.0)$ & 32 \\
$30-39 \mathrm{yr}$ & $53(84.1)$ & $10(15.9)$ & 63 \\
$40-49 \mathrm{yr}$ & $47(81.0)$ & $11(19.0)$ & 58 \\
$50-59 \mathrm{yr}$ & $52(80.0)$ & $13(20.0)$ & 65 \\
$60-69 \mathrm{yr}$ & $40(87.0)$ & $6(13.0)$ & 46 \\
$70-79 \mathrm{yr}$ & $26(96.3)$ & $1(3.7)$ & 27 \\
$80-89 \mathrm{yr}$ & $7(70.0)$ & $3(30.0)$ & 10 \\
$>90 \mathrm{yr}$ & $1(100.0)$ & $0(0.0)$ & 1 \\
\hline Total & $332(88.0)$ & $45(11.9)$ & 377 \\
\hline
\end{tabular}

Values are presented as number (\%).

test results was limited by small sample sizes in certain subgroups. Analyses were conducted using SAS 9.4 software (SAS Institute Inc., Cary, NC, USA). The $P \leq 0.05$ represented a statistically significant result.

\section{Results}

This population size in this study was 377 subjects (754 knees). The average age was $41.22 \pm 21.37$ years with a range of 3 to 100 years old. There were 158 male (41.9\%) and 219 female (58.1\%) subjects.

\section{Fabella}

Out of the 377 subjects, fabella was present in 45 subjects (11.9\%). The mean age of subjects with fabella was 50.39 years (range, 17-84 years), while that of subjects without fabella was 39.95 years. Of these 45 subjects, 23 were male and 22 were female (Table 1). Regarding laterality of fabella, the majority 32 subjects (72.7\%) had bilateral fabella, while the remaining were unilateral as shown in Table 1. Among the 32 subjects with bilateral fabella, 15 (46.9\%) were symmetric 
Table 3. Crosstabulation of fabella diameters among male and female subjects and test of significant difference of the diameters between male and female subjects

\begin{tabular}{clcccccc}
\hline Fabella diameters $(\mathrm{mm})$ & Sex & Number & Mean & SD & $95 \%$ CI & Min-Max & Pr>|t $\mid$ \\
\hline Craniocaudal & Male & 24 & 4.03 & 1.35 & $-0.81-0.77$ & $2.00-7.00$ \\
& Female & 25 & 4.05 & 1.42 & & 0.73 \\
Anteroposterior & Male & 24 & 2.64 & 1.00 & $-0.64-0.78$ & $1.00-6.00$ \\
& Female & 25 & 2.61 & 1.30 & & 0.49 \\
Fabello-femoral & Male & 24 & 4.83 & 2.90 & $-0.46-2.39$ & $0.00-12.00$ \\
& Female & 25 & 3.87 & 2.00 & & 0.24 \\
Fabello-tibial & Male & 24 & 4.79 & 3.18 & $-1.01-1.97$ & $0.60-12.00$ \\
& Female & 25 & 4.31 & 1.86 & & 0.003 \\
\hline
\end{tabular}

Values are presented as number only. SD, standard deviation; CI, confidence interval, Min, minimum; Max, maximum.

Table 4. Crosstabulation of patella configuration with sex

\begin{tabular}{lccccc}
\hline Sex & $\begin{array}{c}\text { Unipartite } \\
\text { bilaterally }\end{array}$ & $\begin{array}{c}\text { Bipartite \& } \\
\text { multipartite } \\
\text { patella }\end{array}$ & $\begin{array}{c}\text { No } \\
\text { patella }\end{array}$ & $\begin{array}{c}\text { Just } \\
\text { ossifying }\end{array}$ & Total \\
\hline Male & 142 & 7 & 8 & 1 & 158 \\
Female & 209 & 1 & 1 & 8 & 219 \\
Total & 351 & 1 & 9 & 9 & 377 \\
\hline
\end{tabular}

in size while 17 (53.1\%) were asymmetric in size. Subjects with fabella were stratified into various age groups (Table 2). As regards laterality of fabella, age category 40 to 49 years had the highest frequency of unilateral fabella. Age category 50 to 59 years had the highest frequency of bilateral fabella followed by the 30 to 39 and 40 to 49 -years categories. Age category 50 to 59 years had the highest frequency among subjects with bilateral fabella symmetric in size. Age category 40 to 49 years had the highest frequency among subjects with bilateral fabella asymmetric in size followed by age 30 to 39 years.

The prevalence of fabella was similar between male and female subjects $(P=0.36$; odds ratio [OR], 0.75 ; $95 \%$ confidence interval [CI], 0.41-1.38). Among the age groups, there was a positive association between age $<47$ and $\geq 47$ and the prevalence of fabella $(P<0.0146)$. Fabella prevalence increased up to approximately age 60 , then decreased abruptly after the $60 \mathrm{~s}$, and increased again in 80 s (Table 2). These results are insignificant and likely due to limitations in sample size within certain age groups. There was no difference between the distribution of fabella symmetry/asymmetry in both knees among male and female subjects, $P=0.49$. There was no between bilateral fabella symmetry/asymmetry among the age categories, $P=0.43$. There was no difference between the laterality of fabella among males and females, $P=0.41$. Although the mean ages of subjects with left, right and bilateral fabella were similar $(50.27,50.40$, and 49.97 years, respectively), the laterality of fabella was different among the various age groups, $P=0.02$.

The mean CC diameter was $4.09 \pm 1.48 \mathrm{~mm}$ (range, $2-9$ $\mathrm{mm}$ ). The mean AP diameter was $2.66 \pm 1.15 \mathrm{~mm}$ (range, $1-6$ $\mathrm{mm}$ ). The mean FFD was $4.38 \pm 2.56 \mathrm{~mm}$ (range, $1-13 \mathrm{~mm}$ ), while the mean FTD was $4.35 \pm 2.47 \mathrm{~mm}$ (range, $0.6-12 \mathrm{~mm}$ ) (Table 3).

\section{Patella variants}

The overall prevalence of bipartite and multipartite patella in this study was $2.1 \%$ (8 subjects). There were seven subjects with bipartite patella and one subject with multipartite patella. There were two subjects with Saupe type I bipartite patella, two subjects with Saupe type II bipartite patella, three subjects had type III bipartite patella and one subject had type III multipartite patella. Among the 377 subjects, 351 subjects had normal Unipartite patella bilaterally, the patellae of nine subjects had yet to ossify, while nine subjects had a 'just ossifying' patella - bone just beginning to be visible on radiograph with the youngest subject in this group being 3 years old (Fig. 5). The laterality and sex distribution of bipartite and multipartite patella are shown in Table 4 .

Among male and female subjects, the difference in prevalence of bi and multipartite patella was statistically significantly ( $P=0.03$; OR, 10.17 ; 95\% CI, 1.24-85.34), with males being significantly more likely to have bi and multipartite patella than females. Among age groups, individuals $\geq 47$ were more likely to have unipartite patella rather than bipartitie or multipartite compared to those $<47$. For the presence of "no patella," there was a statistically significant difference between those $<47$ years old and $47+$ years old, $P \leq 0.0001$.

Evaluating for any association between the incidence of fabella and patella variants, there was no statistically significant association between the prevalence of fabella and patella $(P=0.98$; OR, 0.98; 95\% CI, 0.12-8.11). 


\section{Discussion}

\section{Fabella}

Although there is a consensus that fabella is a normal anatomical variant, it could be involved in clinically significant knee pathologies like fabella fracture, fabella dislocation, fabella syndrome, peroneal nerve impingement, chondromalacia fabellae, and it is also associated with knee osteoarthritis [19, 22, 27-29].

The overall imaging prevalence of fabella in this study $(11.9 \%)$ is on the lower end of the range reported (10\%-30\%) in western Europe among Caucasian populations. In consonance with our findings, a 2019 global meta-analysis also found the lowest prevalence of fabella in Africa [30]. Although this meta-analysis included numerous studies worldwide, it included only one cadaveric study from Africa. Studies among Caucasians in North America have reported a fabella prevalence range of $15.0 \%$ to $31.3 \%$ [21, 23, 24]. Radiographic and magnetic resonance imaging studies in Turkey and Singapore have reported fabella prevalence of $22.8 \%$ and $31.25 \%[14,25]$. The lower prevalence in our study compared to other studies using radiographs could possibly be attributed to the population makeup of our cohort. There is no other imaging study from sub-Saharan Africa that we could compare our findings with. A cadaveric study in South Africa reported a fabella prevalence of $25.3 \%$ which is approximately twice the prevalence in our study [20]. Higher prevalence of fabella in studies using cadaver dissection has been observed in other regions around the world. The highest reported prevalence of fabella in literature have been in studies using cadavers in Asia. A study in Japan found a fabella prevalence of $85.8 \%$ and a study using both cadaver and radiographs in China found a fabella prevalence $86.9 \%$ $[12,31]$. Some authors have postulated that the increased prevalence of fabella in studies using cadaver dissection is due to both cartilaginous fabella and osseous/bony fabella being easily identified on cadaver dissections while radiographs can only detect ossified/bony fabella [12]. A group of Turkish authors also surmised that studies across Asia had higher fabella prevalence compared to western European and North American studies because everyday life in some Asian countries probably requires more mechanistic use of the knee, such a frequent kneeling, which led to increased fabella prevalence in that region [25]. In this study, fabella prevalence increased up to approximately age 60 , then decreased abruptly after the 60s, and increased again in $80 \mathrm{~s}$.
Our results are likely due to limitations in sample size, and small participant numbers within some age groups. While there is a significant difference between the $<47$ and $47+$ age groups, the difference between the $60 \mathrm{~s}, 70 \mathrm{~s}$, and 80 s group is insignificant. This is because the fabella will present increasing frequency as it is formed in younger individuals and then level out in middle age [32].

The prevalence of bilateral fabella in both males and females was twice that of unilateral fabella. This 2:1 ratio was also seen in a study among Caucasians in Turkey [25]. The 2019 global meta-analysis also reported that $72.9 \%$ of the cases studied were bilateral fabella [31]. The prevalence among males and females were approximately equal, similar to a study among central Europeans which showed 1:1 prevalence of fabella in males and females [13]. The cadaveric study in South Africa involving Caucasian and Black African subjects showed a higher male and female prevalence of $21.2 \%$ and $27.8 \%$ compared to this study [20]. As started earlier, the prevalence of fabella is usually higher in cadaveric studies than in radiographic studies. Some studies have found positive correlation between age and prevalence of fabella, while others did not find significant differences in fabella prevalence among various age groups $[31,33]$. It is believed that aging causes an increase in cartilaginous fabella ossification which may account for the increased prevalence in older age groups [25]. There was a positive association between age and occurrence of fabella in this study $(P<0.015)$, which correlates with the theory that aging increases cartilaginous fabella ossification therefore making it visible on radiograph. Accumulation of mechanical stresses from everyday activity over time could also be a factor for why older subjects had a higher prevalence of fabella in this study. Since there is a reported positive correlation between mechanobiological factors and increased fabella ossification [32].

The relative size of fabella in bilateral cases and its proximity to the common fibular (CF) nerve and popliteal artery could be a predictor of CF nerve palsy, Popliteal Artery Entrapment Syndrome and other symptoms [10]. Among the 32 subjects with bilateral fabella, the frequency of symmetric and asymmetric fabella was approximately equal with no statistically significant association between the prevalence of symmetric and asymmetric fabella among sex, and age groups. To the best of our knowledge, there is no study in literature comparing the sizes of bilateral fabella, so we are not able to compare this finding with any previous study.

Linear dimensions of fabella have been reported in litera- 
ture. Fabella lengths in the range of 4 to $22 \mathrm{~mm}$ have been recorded in symptomatic patients $[12,34]$. In this study, the CCD (length) and APD (depth/thickness) of the fabella in males $(4.05 \pm 1.38 \mathrm{~mm}, 2.75 \pm 1.03 \mathrm{~mm}$, respectively) and females $(4.12 \pm 1.57 \mathrm{~mm}, 2.58 \pm 1.27 \mathrm{~mm}$, respectively) were shorter compared to the recorded values among an Asian cohort in Singapore. Chew et al. [14] reported that male fabella had a median length of $7.50 \mathrm{~mm}$ and width of $6.95 \mathrm{~mm}$ while female fabella had a median length of $5.86 \mathrm{~mm}$ and width of $5.49 \mathrm{~mm}$. Another study in the United States reported an average fabella length of $7.9 \mathrm{~mm}$ and width of $4.2 \mathrm{~mm}$ [11]. This discrepancy could be attributed to the different populations involved in this study and the other two studies since genetic and geographical factors play a significant role in fabella prevalence and ossification. In other words, it is conceivable that fabellas are smaller in this African population than elsewhere. Despite the numerical differences, this study and the Singaporean study did not find any statistically significant difference in the length and width of fabella between males and females [14].

There is a reported association between very small and very large (FTD) and presence of Baker's cysts in the knee. This is because Baker's cyst can displace the fabella toward the tibia causing a very small FTD or displace the fabella away from the femur causing a very large FTD [11]. Friedman and Naidich [11] reported an FTD average value of $14.0 \pm 3.5 \mathrm{~mm}$ in their cohort. The average FTD in males $(4.50 \pm 3.19 \mathrm{~mm})$ and females $(4.23 \pm 1.79 \mathrm{~mm})$ in this study is lower than the one reported by Friedman and Naidich [11]. Also, since there is a paucity of data in the literature reporting the average FTD values among various populations, the difference in values reported by both studies could be due to intrinsic differences between the two populations.

Patients with synovial effusion and pigmented villonodular synovitis have been reported to have a very large FFD [11]. This association has been reported to be due to posterior displacement of the fabella causing a large increase in FTD. Using the FFD measurements in patients with fabella is advantageous since it can be used even in technically unsatisfactory radiographic scans unlike the fat pad separation sign [35]. Therefore, knowing the reference values for FFD and FTD in this population could be clinically relevant to radiologists, orthopedic surgeons, and other clinicians in managing various knee pathologies in patients of Black African descent. At the time of acquiring the radiographs in this study, none of the subjects in this study were confirmed to have any knee pathologies such as effusions, synovial masses, or Baker's cyst. It is worth noting, that more studies among populations of Black African descent are needed to fully ascertain what the normal range of FTD and FFD should be in this population.

\section{Patella variants}

The overall prevalence of bipartite and multipartite patella in this study was $2.1 \%$. A study in the United States found a bipartite patella prevalence of $12.5 \%$ [9]. Bipartite and multipartite patella was significantly more likely to be found in males than females in this study cohort, $P=0.03$. This significant difference was similar to a study in the United States where $76 \%$ of all patients with bipartite patella were male and to a Japanese study using radiographs in which $77 \%$ of all patients with bipartite patella were male [7, 9]. Among the subjects with bipartite and multipartite patella, $62.5 \%$ were below 40 years. This finding along with the increased prevalence in males could explain the findings in other studies that symptomatic bipartite and multipartite patella occur more in younger males although none of the subjects in this study had reported any symptoms of knee pain during radiography $[7,9]$. It is worth noting that among the age groups in this study cohort, there was no significant difference between the prevalence of bipartite and multipartite patella with $P=0.98$.

Congenital absence of patella is rare and is usually among the findings of the hereditary Nail Patella Syndrome, ischiopatellar dysplasia or orthopedic anomalies [36, 37]. There were 9 subjects $(2.4 \%)$ evaluated to have had 'no patella' with all 9 subjects' ages ranging from 3 to 4 years old. This implies that ossification of patella in our study population may not start at 3 years as observed in Caucasians. Understanding the age of patella ossification in this population could help radiologist in identifying patients of Black African descent with anomalies involving absent patella or delayed ossification of patella.

\section{Fabella and patella variants}

Fabella and patella variants are both sesamoid bones found in the knee area, therefore it is imperative to evaluate their relative prevalence. In this study, the presence of fabella was not significantly associated with the presence of bipartite and multipartite patella, $P=0.10$. This implies there may be no link between a patient having fabella and patella variants. Since there is no other study in literature that evaluates this 
association, studies with higher statistical power are needed to conclusively determine the co-occurrence of fabella and patella variants.

This study utilized plain radiographs in evaluating fabella, so we were only able to evaluate bony fabella and not cartilaginous fabella. This could have lowered the prevalence of fabella in this study. Due to the retrospective nature of this study, we were unable to examine how anthropometric factors could have affected the prevalence and distribution of fabella and patella variants in this population.

The prevalence of fabella and patella variants were lower in this study compared to the findings in other populations and ethnicities. Male subjects tended to have higher prevalence of bipartite and multipartite patella. Understanding the findings and relationships presented in this study could be clinically useful to radiologists, orthopedic surgeons, and other healthcare practitioners in managing patients of African descent presenting with knee joint pathologies. This study also provides preliminary data for future studies of fabella and patella variants and their association with knee pathologies and clinical presentations. Such studies would further guide clinical management of symptomatic patients of Black African descent with fabella and patella variants.

\section{ORCID}

Joseph Adetola Adedigba:

https://orcid.org/0000-0003-1172-2896

Bukunmi Michael Idowu:

https://orcid.org/0000-0002-9811-5644

Sarah Perou Hermans:

https://orcid.org/0000-0001-5129-7914

Onoja Frederick Okwori:

https://orcid.org/0000-0002-1271-3433

Stephen Olaoluwa Onigbinde:

https://orcid.org/0000-0002-6179-3505

Kehinde S. Oluwadiya:

https://orcid.org/0000-0002-4277-4384

Angela Ameyaa Amoako:

https://orcid.org/0000-0003-0503-5846

Mandy C. Weidenhaft:

https://orcid.org/0000-0002-3719-1643

\section{Author Contributions}

Conceptualization: BMI, SOO. Data acquisition: JAA,
BMI. Data analysis or interpretation: JAA, SPH, OFO, KSO, AAA. Drafting of the manuscript: JAA, BMI, SPH, AAA. Critical revision of the manuscript: JAA, BMI, KSO, MCW. Approval of the final version of the manuscript: all authors.

\section{Conflicts of Interest}

No potential conflict of interest relevant to this article was reported.

\section{References}

1. Eyal S, Rubin S, Krief S, Levin L, Zelzer E. Common cellular origin and diverging developmental programs for different sesamoid bones. Development 2019;146:dev167452.

2. Seguritan RE, Wolfe AR, Mena P, Bibawy J, Bianchi C, Solomon N, Kikkeri V. Bipartite patella separation and partial quadriceps tendon rupture in the setting of trauma. Radiol Case Rep 2018;14:526-9.

3. Sarkodieh J, Gobindpuri A. The age of patella ossification [Internet]. Vienna: European Society of Radiology; c2016 [cited 2020 May 7]. Available from: https://epos.myesr.org/esr/viewing/index.php?module=viewing_posteraction\&task=downloa dpdf\&pi=135454.

4. Zabierek S, Zabierek J, Kwapisz A, Domzalski ME. Bipartite patella in 35-year-old fitness instructor: a case report. Int J Sports Phys Ther 2016;11:777-83.

5. Gruber W. [In Bildungsanomalie mit Bildungshemmung begründete Bipartition beider Patellae eines jungen Subjectes]. Arch Pathol Anat 1883;94:358-361. German.

6. Saupe E. Beitrag zur Patella bipartite. Fortschr Rontgenstr 1921;28:37-41.

7. Oohashi Y, Koshino T, Oohashi Y. Clinical features and classification of bipartite or tripartite patella. Knee Surg Sports Traumatol Arthrosc 2010;18:1465-9.

8. Perdikakis E, Pothitakis C. Symptomatic multipartite patella: imaging findings and pain relief. A description of two cases. Cent Eur J Med 2013;8:172-175.

9. Omar IM, Schweitzer M. Bipartite patella: MR imaging characteristics and frequency. Proc Intl Soc Mag Reson Med 2004;11:804.

10. Dalip D, Iwanaga J, Oskouian RJ, Tubbs RS. A comprehensive review of the fabella bone. Cureus 2018;10:e2736.

11. Friedman AC, Naidich TP. The fabella sign: fabella displacement in synovial effusion and popliteal fossa masses. Normal and abnormal fabello-femoral and fabello-tibial distances. Radiology 1978;127:113-21.

12. Minowa T, Murakami G, Kura H, Suzuki D, Han SH, Yamashita T. Does the fabella contribute to the reinforcement of the posterolateral corner of the knee by inducing the development of associated ligaments? J Orthop Sci 2004;9:59-65.

13. Hauser NH, Hoechel S, Toranelli M, Klaws J, Müller-Gerbl M. 
Functional and structural details about the fabella: what the important stabilizer looks like in the central European population. Biomed Res Int 2015;2015:343728.

14. Chew CP, Lee KH, Koh JS, Howe TS. Incidence and radiological characteristics of fabellae in an Asian population. Singapore Med J 2014;55:198-201.

15. Franceschi F, Longo UG, Ruzzini L, Leonardi F, Rojas M, Gualdi G, Denaro V. Dislocation of an enlarged fabella as uncommon cause of knee pain: a case report. Knee 2007;14:330-2.

16. Agathangelidis F, Vampertzis T, Gkouliopoulou E, Papastergiou S. Symptomatic enlarged fabella. BMJ Case Rep 2016;2016:bcr2016218085.

17. Heideman GM, Baynes KE, Mautz AP, DuBois MS, Roberts JW. Fabella fracture with CT imaging: a case report. Emerg Radiol 2011;18:357-61.

18. Theodorou SJ, Theodorou DJ, Resnick D. Painful stress fractures of the fabella in patients with total knee arthroplasty. AJR Am J Roentgenol 2005;185:1141-4.

19. Driessen A, Balke M, Offerhaus C, White WJ, Shafizadeh S, Becher C, Bouillon B, Höher J. The fabella syndrome- a rare cause of posterolateral knee pain: a review of the literature and two case reports. BMC Musculoskelet Disord 2014;15:100.

20. Phukubye P, Oyedele O. The incidence and structure of the fabella in a South African cadaver sample. Clin Anat 2011;24:8490.

21. Pritchett JW. The incidence of fabellae in osteoarthrosis of the knee. J Bone Joint Surg Am 1984;66:1379-80.

22. Takebe K, Kita K, Hirohata K. Radiological and anatomical observation on fabella. Orthop Surg 1983;34:1163-70.

23. Sarin VK, Erickson GM, Giori NJ, Bergman AG, Carter DR. Coincident development of sesamoid bones and clues to their evolution. Anat Rec 1999;257:174-80.

24. Yu JS, Salonen DC, Hodler J, Haghighi P, Trudell D, Resnick D. Posterolateral aspect of the knee: improved MR imaging with a coronal oblique technique. Radiology 1996;198:199-204.

25. Egerci OF, Kose O, Turan A, Kilicaslan OF, Sekerci R, KelesCelik N. Prevalence and distribution of the fabella: a radio- graphic study in Turkish subjects. Folia Morphol (Warsz) 2017;76:478-83.

26. Er A, Murphy A. Knee (horizontal beam lateral view) [Internet]. Radiopaedia; 2016 [cited 2020 May 7]. Available from: https:// radiopaedia.org/articles/knee-horizontal-beam-lateral-view-1.

27. Zhou F, Zhang F, Deng G, Bi C, Wang J, Wang Q, Wang Q. Fabella fracture with radiological imaging: a case report. Trauma Case Rep 2017;12:19-23.

28. Hou W, Xu L, Wang J, Wang B, Liu L, Xu K, Cai Y, Guo H, Xu P. Fabellar prevalence, degeneration and association with knee osteoarthritis in the Chinese population. Sci Rep 2019;9:13046.

29. Pop TS, Pop AM, Olah P, Trâmbiţaş C. Prevalence of the fabella and its association with pain in the posterolateral corner of the knee: a cross-sectional study in a Romanian population. Medicine (Baltimore) 2018;97:e13333.

30. Berthaume MA, Bull AMJ. Human biological variation in sesamoid bone prevalence: the curious case of the fabella. J Anat 2020;236:228-42.

31. Zeng SX, Dong XL, Dang RS, Wu GS, Wang JF, Wang D, Huang HL, Guo XD. Anatomic study of fabella and its surrounding structures in a Chinese population. Surg Radiol Anat 2012;34:65-71.

32. Tabira Y, Saga T, Takahashi N, Watanabe K, Nakamura M, Yamaki K. Influence of a fabella in the gastrocnemius muscle on the common fibular nerve in Japanese subjects. Clin Anat 2013;26:893-902.

33. Takebe K, Hirohata K. Peroneal nerve palsy due to fabella. Arch Orthop Trauma Surg 1981;99:91-5.

34. Mangieri JV. Peroneal-nerve injury from an enlarged fabella. A case report. J Bone Joint Surg Am 1973;55:395-7.

35. Hall FM. The fabella sign and radiologic assessment of knee joint effusion. Radiology 1978;129:541-2.

36. Jerome JT, Varghese M, Sankaran B, Thomas S. Bilateral congenital absence of patella. Indian J Orthop 2008;42:228-30.

37. Sferopoulos NK. Congenital aplasia of the patella. Int J Radiol 2018;5:172-176. 